\title{
Analysis of Ultrastructural Properties Reveals SP-D Protective Roles in Lung and Pancreatic Injury of Severe Acute Pancreatitis in Mouse Model
}

\author{
Han Chen ${ }^{1}$, Jia Yu ${ }^{2}$ and Guirong Wang ${ }^{2}$ \\ 1. Microscopy Core Facility, Department of Research Resource, The Pennsylvania State University, \\ College of Medicine, Hershey, PA, USA. \\ 2. Department of Surgery, SUNY Upstate Medical University, Syracuse, NY, USA.
}

Severe acute pancreatitis (SAP) often causes significant multiple organ (i.e. lung and pancreas) injury by an inflammatory response with the involvement of a cascade of cellular and molecular events ${ }^{1}$. Surfactant protein D (SP-D), an innate immune molecule, plays critical roles in host defense and the regulation of inflammation ${ }^{2}$. Our previous study has demonstrated that SP-D had a protective effect on the pancreatic injury in the cecal ligation and puncture (CLP)-induced septic murine model ${ }^{3}$. The NLRP3 inflammasomes and nuclear factor kappa B (NF- $\mathrm{B})$ signaling molecules are key regulators of innate immunity and inflammatory response. We hypothesized that SP-D can attenuate inflammation and tissue injury by suppressing NLRP3 inflammasome activation and regulating NF- $\kappa$ B signaling pathway in experimental SAP. In order to reveal the mechanisms in the pathogenesis of SAP and the SP$\mathrm{D}$ effect we performed the detailed analyses of ultrastructural properties of lung and pancreas using transmission electron microscopic technique in this specific project.

Wild-type C57BL/6 (WT), SP-D knockout (KO), and humanized transgenic SP-D (hTG, with lungspecific human SP-D expression without mouse SP-D background) mice were used in this study. SAP was induced by both administration of lipopolysaccharide (LPS, $10 \mathrm{mg} / \mathrm{kg} . \mathrm{BW}$ ) and 6 hourly intraperitoneal injections of caerulein $(100 \mu \mathrm{g} / \mathrm{kg}$.BW). Animals were sacrificed 1 and 18 hours after the last caerulein treatment. Histopathologic changes in lung, pancreatic and liver tissues were assessed by $\mathrm{H} / \mathrm{E}$ staining and light microscopy. Ultrastructural changes (autophagy, cellular and organelle injury) were analyzed using transmission electron microscope (EM). The lung and pancreatic tissues were fixed by $2.5 \%$ glutaraldehyde and $2 \%$ paraformaldehyde in $0.1 \mathrm{M}$ phosphate buffer $(\mathrm{pH} 7.4)$ and further fixed in $1 \%$ osmium tetroxide in $0.1 \mathrm{M}$ phosphate buffer $(\mathrm{pH} 7.4)$ for 1 hour. Samples dehydrated in a graduated ethanol series, and embedded in LX-112 (Ladd Research Industries, Vermont, VA). Thin sections $(70 \mathrm{~nm})$ were cut by Diatome knife and stained with uranyl acetate and lead citrate and viewed in a JEOL JEM1400 Transmission Electron Microscope (JEOL USA Inc., Peabody, MA, USA). In addition, the NLRP3 inflammasome, NF- $\mathrm{B}$ activation and apoptosis in lungs and pancreases were analyzed by western blotting, immunofluorescence and TUNEL methods. It is considered statistical significance when $\mathrm{p}<0.05$.

The results demonstrated that treatment with both caerulein and LPS caused multiple organ injury including lung and pancreas in all three types of (WT, KO and hTG) mice. SP-D KO mice showed more severe pancreas and lung injury compared to WT mice $(P<0.05)$. hTG mice exhibited similar lung injury as WT mice. The ultrastructural abnormalities of mitochondria, severe rough endoplasmic reticulum (rER) damage and reduced number of ribosomes, and significant autophagosome formation are found in SP-D KO mice pulmonary endothelial and epithelial cells (Fig. 1). These abnormalities consist not only of a decrease of the number of mitochondria but also of an enlarged and abnormal shape, variations in the number of cristae and particular patterns of cristae, and abnormal inclusions. The functional consequences of these mitochondrial abnormalities can be far reaching and systemic due to the common 
underlying impairment of oxidative phosphorylation. In Fig 2, a detail from a pulmonary epithelial cell is shown abnormal lamellar bodies. EM analysis revealed that there are significant differences of autophagosome formation, mitochondrial and rough endoplasmic reticulum (rER) injury, and the number of ribosome bodies in the lung type II cells and pancreatic acinar cells between SAP KO and SAP KO (SAP) mice. Furthermore, SAP mice exhibited severe rough endoplasmic reticulum (rER) damage and reduced number of ribosomes, and significant autophagosome formation in pancreatic acinar cells (Fig. 3 and 4); and damaged lamellar bodies (LBs) with decreased number of LBs in the lung AT II cells, when compared to control mice. These provided direct evidence of severe pancreatic and lung injury in the SAP model.

These results demonstrated that SP-D plays a protective role of in the SAP-induced multiple organ injury through regulating innate inflammation, autophagy, mitochondria and rER dysfunction and apoptosis [4].

\section{References:}

[1] E Charbonney and AB Nathens, Surg Infect (Larchmt) 9 (2008), p. 573.

[2] JR Wright, Nat Rev Immunol 5 (2005), p. 58.

[3] Z Liu et al., Sci Rep 5 (2015), p. 17798.

[4] The authors acknowledge funding from NIH R01 HL136706 and NSF research award 172263.

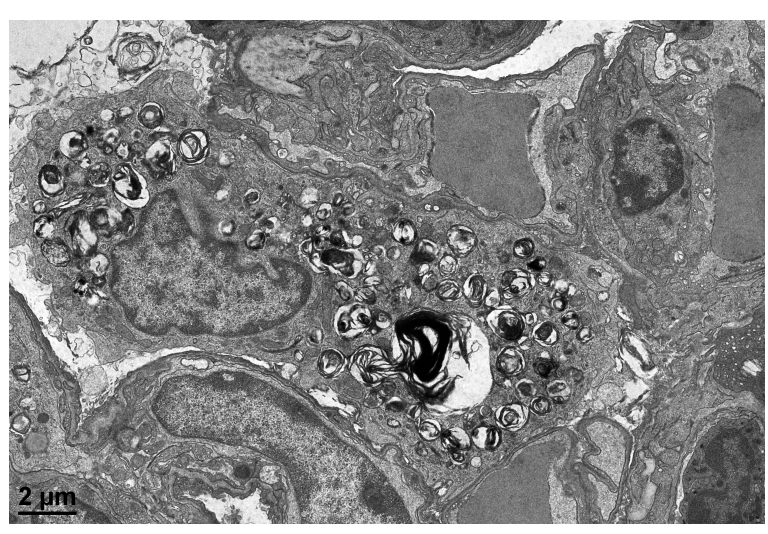

Figure 1. SP-D KO (SAP) mice pulmonary cell. Scale bar 2 um

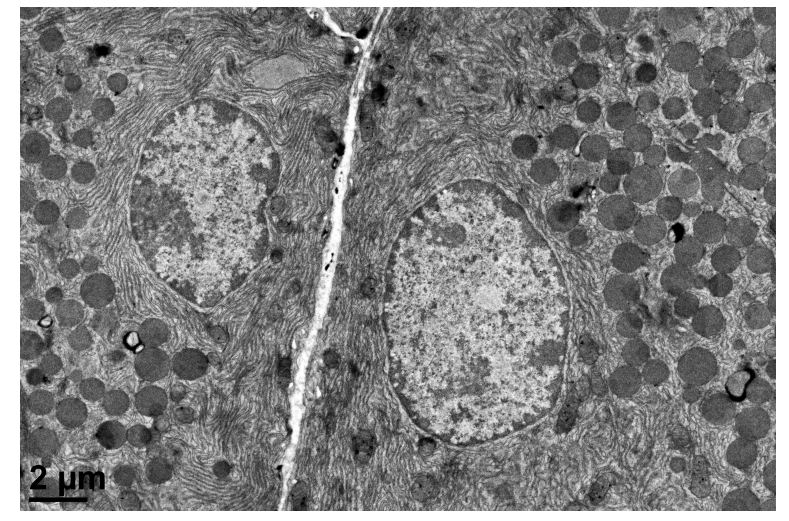

Figure 3. SP-D KO mice pancreatic acinar cells Scale bar 2 um

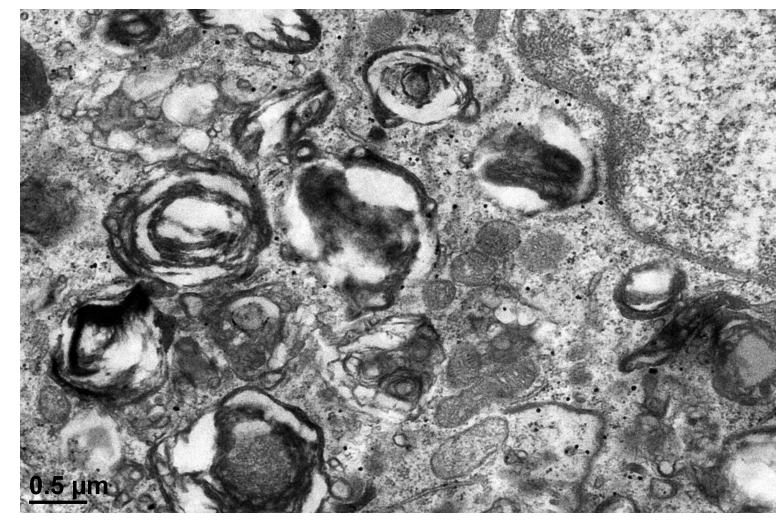

Figure 2. SP-D KO mice (SAP) pulmonary cell. Scale bar 0.5 um.

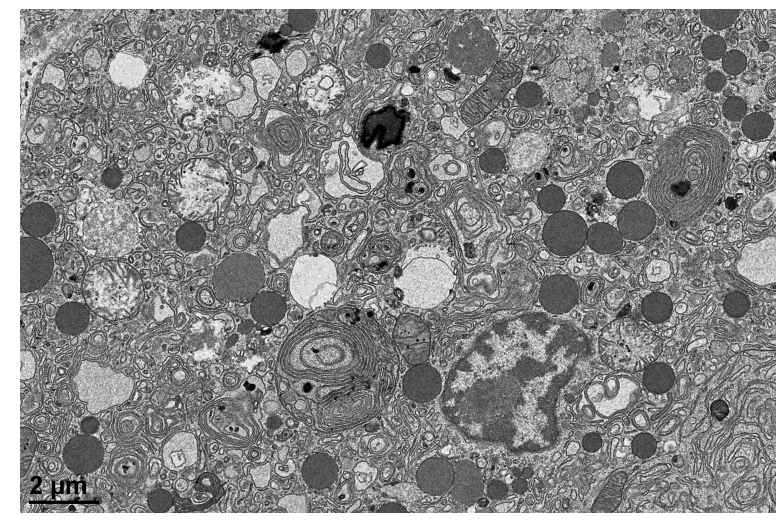

Figure 4. SP-D KO(SAP) mice pancreatic acinar cells Scale bar 2 um 